Gefässchirurgie $2021 \cdot 26: 337-338$

https://doi.org/10.1007/s00772-021-00805-w

Angenommen: 14. Juli 2021

C) Springer Medizin Verlag $\mathrm{GmbH}$, ein Teil von Springer Nature 2021

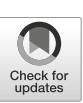

\section{Faszination Gefäßchirurgie: innovativ und offen}

\author{
Markus Steinbauer
}

Klinik für Gefäßchirurgie, Krankenhaus Barmherzige Brüder Regensburg, Regensburg, Deutschland
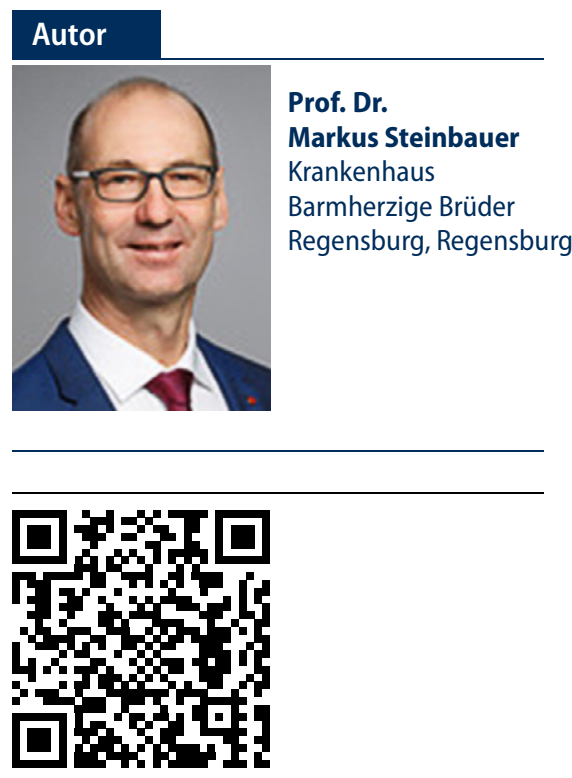

QR-Code scannen \&Beitrag online lesen
Unter dem Motto „Faszination Gefäßchirurgie: innovativ und offen" findet vom 13.10.-16.10.2021 die 37. Jahrestagung der Deutschen Gesellschaft für Gefäßchirurgie und Gefäßmedizin in Mannheim statt. Entsprechend diesem positiven und zukunftsgewandten Motto möchten wir auch in diesem Themenheft die Faszination der Gefäßchirurgie sowie deren Innovationskraft und Offenheit im interdisziplinären Austausch aus verschiedenen Perspektiven darstellen.

Frau Dr. Katrin Meisenbacher (Heidelberg) stellt aus der Sicht einer jungen engagierten Gefäßchirurgin dar, aus welchen Gründen sie dieses Fach gewählt hat und was sie für die Zukunft von der Weiterentwicklung des Faches Gefäßchirurgie erwartet.

Prof. Dr. H.-H. Eckstein (München), einer der prägenden akademischen Gefäßchirurgen Europas, berichtet rückschauend über seine Sicht der Faszination und Entwicklung der Gefäßchirurgie in Deutschland.

Entscheidend für die weitere wissenschaftliche und klinische Entwicklung der Gefäßchirurgie ist eine ungebrochene In- novationskraft in der endovaskulären Therapie, gepaart mit dem Erhalt und der Modifikation der offenen gefäßchirurgischen Techniken.

\section{॥ Entscheidend für die}

Gefäßchirurgie ist ungebrochene Innovationskraft in der offenen und endovaskulären Therapie

In diesem Sinne skizziert Dr. Tessarek (Lingen) die Innovationen und die revolutionären Entwicklungen für die in der Gefäßchirurgie so wichtige endovaskuläre Therapie der peripheren arteriellen Verschlusserkrankung.

Die offen-chirurgische Behandlung von Gefäßerkrankungen stellt ein Alleinstellungsmerkmal der Gefäßchirurgie in der Behandlung von Gefäßpatienten dar. Umso wichtiger ist eine Perspektive, wie sich die offene Gefäßchirurgie innovativ und patientenorientiert in der Zukunft weiterentwickeln kann. Herr A. Arnautovic (Düsseldorf) stellt hier mögliche Szenarien für die Zukunft dar.

"Innovativ und offen" bedeutet auch offen zu sein für Entwicklungen bezüglich 
der kommenden Herausforderung durch eine alternde Gesellschaft. Aufgrund der immer älter werdenden Patienten sind Überlegungen zur Implementierung von geriatrischen Versorgungskonzepten in der Gefäßmedizin und Gefäßchirurgie zwingend notwendig, um auch in der Zukunft eine qualitativ hochwertige und patientenzentrierte Versorgung leisten zu können. B. Maassen (Aachen) stellt mögliche geriatrische Konzepte für die Gefäßmedizin und Gefäßchirurgie dar.

Eine ganzheitliche und umfassende Versorgung von Gefäßpatienten aus einer Hand beinhaltet auch, dass in Gefäßzentren und gefäßchirurgischen Einheiten eine palliativmedizinische Versorgung implementiert und angeboten werden sollte.

Frau Dr. Neuwert (Regensburg) stellt wichtige Säulen der Palliativmedizin vor, die zunehmend auch für gefäßchirurgische/-medizinische Patienten immer mehr an Bedeutung gewinnen werden.

Das Themenheft möchte Denkanstöße geben und mögliche Innovationen für die Entwicklung der Gefäßchirurgie und Gefäßmedizin skizzieren und wir wünschen Ihnen bei der Lektüre viel Freude an der „Faszination Gefäßchirurgie".

Wir würden uns freuen, wenn wir Sie zahlreich auf der 37. Jahrestagung der Deutschen Gesellschaft für Gefäßchirurgie in Mannheim oder im digitalen Hybridformat begrüßen könnten.

Ihr

Prof. Dr. med. Markus Steinbauer

Präsident der Deutschen Gesellschaft für Gefäßchirurgie und Gefäßmedizin (DGG e.V.)

Kongresspräsident der 37. Jahrestagung der DGG 2021

\section{Korrespondenzadresse}

\section{Prof. Dr. Markus Steinbauer}

Klinik für Gefäßchirurgie, Krankenhaus

Barmherzige Brüder Regensburg

Prüfeninger Straße 86, 93049 Regensburg,

Deutschland

markus.Steinbauer@barmherzige-

regensburg.de

Interessenkonflikt. M. Steinbauer gibt an, dass kein Interessenkonflikt besteht.

\section{Umweltverschmutzung durch Plastik}

Neuer Arbeitskreis Plastik und Nachhaltigkeit der Deutschen

Dermatologischen Gesellschaft startet Initiative - Vernetzung mit anderen Fachgruppen angestrebt

Der gesamte Gesundheitssektor wird täglich mit Verpackungsmaterialien aus Kunststoffen unterschiedlichster Art geflutet. Im medizinischen Bereich notwendig, um gesetzlich vorgeschriebene Hygienestandards zu erfüllen, stellt die zunehmende Umstellung auf Einmalartikel wie z.B.OP-Besteck und auch die in der Pandemie verwendeten Masken und Kittel ein wachsendes Entsorgungsproblem dar. Dabei ist die Umweltverschmutzung durch Plastik längst zu einer der schwierigsten Herausforderungen unserer Zeit geworden.

Zudem sind Kunststoffe künstlich erschaffene Rohstoffe vielfältigster Art aus Erdöl und Erdgas. Die weltweite Kunststoffproduktion macht aktuell 10 bis $13 \%$ des gesamten Kohlendioxidanteils am Maximalbudget zur Erhaltung des 1,5-Grad-Zieles der Erderwärmung bis 2050 aus. Insgesamt ist der Gesundheitssektor für etwa $5 \%$ der Treibhausgasemissionen verantwortlich und trägt damit aktiv zur Klimakrise bei.

Um die globale Umweltverschmutzung und die Treibhausgase durch Plastik zu reduzieren, muss der Gesundheitssektor auf den Prüfstand gestellt werden. Gerade von dermatologischen Kliniken und Praxen werden regelmäßig Lokaltherapien empfohlen oder verordnet, die möglicherweise gesundheitsschädliche Stoffe enthalten. Winzige Plastikpartikel unterschiedlichster chemischer Zusammensetzung - Mikroplastik und flüssige Polymere - sind zudem biologisch nicht oder nur schwer abbaubar, sie akkumulieren in der Umwelt und gelangen durch die Nahrungskette zurück auf unseren Teller. Anfang 2020 wurde deshalb der Arbeitskreis Plastik und Nachhaltigkeit in der Dermatologie unter dem Dach der Deutschen Dermatologischen Gesellschaft (DDG) gegründet. Der Arbeitskreis verfügt seit April 2021 über eine mehrsprachige Homepage (www.akdermaplastik.de), die relevante Informationen für Mediziner aller Fachrichtungen zu bedenklichen Inhaltsstoffen in Cremes und Pflegeprodukten bündelt. Zudem vernetzt er sich bundesweit mit nachhaltigen Initiativen aus dem Gesundheitssektor, fördert die Bildung ähnlicher Initiativen in anderen medizinischen Fachrichtungen, fokussiert auf Lösungsvorschläge, indem er Qualitätsmanagement (QM)-Vorlagen für die Transformation zur nachhaltigen Praxis erarbeitet und Patienteninformationen zum Download anbietet.

Ärzt*innen aller Fachrichtungen werden aufgerufen, durch die Umsetzung ressourcenschonender Maßnahmen in der Praxis oder Klinik als Multiplikator*innen einen wichtigen Beitrag zur Aufklärung der Bevölkerung, damit zum Umweltschutz und zum Erhalt der Gesundheit eines jeden Individuums zu leisten.

Interessent*innen und Initiativen können sich gerne unter office@akdermaplastik.de melden.

\author{
Arbeitskreis Plastik und Nachhaltigkeit \\ in der Dermatologie (DDG) \\ Dr. med. Dipl. Biol. Susanne Saha (1. \\ Vorsitzende) \\ Fachärztin für Dermatologie \\ Hautärzte am Marktplatz \\ Kaiserstr. 72 \\ 76133 Karlsruhe
}

Quelle: www.akdermaplastik.de 